\title{
Lidil
}

Revue de linguistique et de didactique des langues

\section{Pour une description linguistique du Mémoire professionnel universitaire}

\author{
Guy Achard-Bayle
}

\section{(2) OpenEdition}

Journals

Édition électronique

URL : http://journals.openedition.org/lidil/21

DOI : $10.4000 /$ lidil.21

ISSN : $1960-6052$

Éditeur

UGA Éditions/Université Grenoble Alpes

\section{Édition imprimée}

Date de publication : 1 décembre 2006

ISBN : 2-914176-15-5

ISSN : 1146-6480

\section{Référence électronique}

Guy Achard-Bayle, «Pour une description linguistique du Mémoire professionnel universitaire », Lidil

[En ligne], 34 | 2006, mis en ligne le 07 avril 2008, consulté le 02 mai 2019. URL : http:// journals.openedition.org/lidil/21 ; DOI : 10.4000/lidil.21

Ce document a été généré automatiquement le 2 mai 2019.

(c) Lidil 


\title{
Pour une description linguistique du Mémoire professionnel universitaire
}

\author{
Guy Achard-Bayle
}

Le mémoire professionnel universitaire :

approche linguistique d'un genre discursif

1 Le mémoire professionnel universitaire (désormais MPU) est un outil de formation (et d'évaluation) théorique et pratique, qui vient sanctionner les cycles d'études professionnelles ou professionnalisantes à BAC +5 (ex-DESS, masters). Il peut être approché de divers points de vue, autrement dit de manière pluri- ou transdisciplinaire ; nous nous y sommes essayé pendant quelques années avec trois autres enseignantschercheurs d'universités littéraire, scientifique et de technologie (Demaizière et Uebersfeld, 2002).

2 Nous nous sommes attaché pour notre part aux aspects linguistiques et plus particulièrement énonciatifs. En cela, le MPU est un genre d'écrit universitaire particulier car il doit permettre au lecteur (et notamment à l'évaluateur) de faire la part et le lien des connaissances et des compétences que l'auteur a acquises aussi bien en théorie qu'en pratique (si l'on admet que c'est l'équilibre des deux et plus encore leur intrication qui caractérise ce type de formation professionnalisante à Bac + 5).

3 Il en résulte, au plan des marques autoréférentielles, que l'auteur est pris entre un nous académique et un je personnel; mais si l'on considère qu'à ce plan personnel, l'intervention d'un moi professionnel implique la présence des autres, et l'interaction de tous, alors le rédacteur se partage entre un je individuel et un nous collectif. En termes d'ancrages personnel mais aussi temporel, cette mise en relation de connaissances et de compétences universitaires et professionnelles et cette interaction entre sujets, entre agents, donnent lieu à un récit (en partie) de type autobiographique. De ce fait, le lecteurévaluateur du MPU, surtout s'il a été directeur, est plus étroitement impliqué dans ce texte qu'il ne l'est dans le cas d'un mémoire scientifique : il n'est plus seulement inclus dans le nous des conventions universitaires, il peut être engagé dans les faits que le récit 
restitue; il ne saurait y être indifférent, même s'il est en désaccord fondamental ou théorique avec l'auteur.

4 Nous voudrions poursuivre ici notre analyse linguistique du MPU (Achard-Bayle, 2004). Comme nous l'avons dit plus haut, notre cadre d'analyse sera la linguistique énonciative'. Dans une première partie, nous chercherons à caractériser génériquement le MPU par l'étude de marques personnelles et spatio-temporelles. Dans la deuxième partie, nous nous attacherons à dépouiller plus systématiquement les occurrences de ces marques dans un MPU en particulier. Nous nous appuyons sur des exemples authentiques, mais, pour les raisons d'implication que nous venons d'évoquer, nous devrons les anonymer.

Le genre MPU du point de vue énonciatifPersonne et pronoms personnels

5 Si la première personne est une marque distinctive du MPU, elle est d'abord celle, singulière, du (réd)acteur, que l'on peut définir comme un double sujet de référence (Achard-Bayle, 2004) : comme le sujet qui fait référence à soi (à son expérience dans le monde) et comme le « centre de perspective sur [c]e monde » (Ricœur, 1990 : 65, suivant Granger, 1979). Je est donc un particulier (1), mais en tant qu'acteur du monde, ou en tant que membre d'une collectivité, il peut être inclus dans un nous effectivement pluriel (2) :

(1) Mon stage dans le cadre du DESS XX s'est déroulé de janvier à juin 1998 à NN Paris.

(2) [Introduction du mémoire] L'échange mondial, et plus particulièrement l'échange à l'intérieur de l'Europe, s'améliore en permanence. Le marché commun, l'ouverture des frontières, le rapprochement des bourses et bientôt la monnaie unique n'en représentent que quelques-unes des nombreuses preuves. Dans un monde qui se rapproche, la communication est autant une cause qu'une conséquence. C'est grâce à un meilleur échange que nous nous rapprochons des autres.

6 Autrement dit, je est un locuteur en même temps que je parle de lui et par là se met à distance. Il y a donc une intrication complexe du locuteur et de l'acteur dans ce je qui est à la marge du monde et dans le monde; ceci oblige le (réd)acteur à de multiples variations sur les personnels : je, nous, on 医 nous y reviendrons dans la seconde partie.

Narration et mémoire

7 Il y a dans "MPU », le terme et la notion de mémoire; ce qui veut dire qu'on peut s'attendre à lire, du moins en partie comme on l'a dit, un texte narratif dont les caractéristiques sont le temps et la présence d'un sujet qui agit par rapport à d'autres actants. De ce point de vue, le MPU que nous avons pris comme exemple est un parfait exemple du genre narratif, en ce que son (réd) acteur tient le rôle du personnage central censé faire évoluer un état de choses (voir la suite de [1]) :

(3) Mon stage [...] avait pour objet l'optimisation (ou plutôt la «mise en marche à partir de zéro ", d'après les termes de $\mathrm{M}^{\mathrm{me}} \mathrm{X}$ ) du centre de ressources multimédia de cet établissement.

Il s'agit donc bien pour l'auteur-acteur de rendre compte d'un processus de transformation, de se situer dans ce processus, et si possible de se représenter comme l'agent principal de ce processus : ce sont autant de points positifs, dans la perspective de l'évaluation, que permet le récit, ou plus exactement la «mise en intrigue » narrative (Ricœur, 1983-1985, 1986, 1990).

Il y a là une première et une grande différence avec le mémoire scientifique. Mais ce qui rapproche encore le MPU des genres narratifs, c'est que ceux-ci font alterner la première 
et la troisième personnes ; on pourra voir les effets, inverses, de ces modalités narratives dans l'extrait suivant :

(4) Il a été décidé de la possibilité d'installer des centres de ressources en entreprise, et d'assurer la permanence dans le centre ainsi que les parties face à face de la formation. Une première expérience dans ce cadre a pu être menée à YY. Il était prévu que je sois intimement liée à ce projet, ce qui n'a en définitive pas été le cas ; j'ai au contraire été écartée de toutes les décisions qui ont été prises. Par ce fait, je n'ai malheureusement pas eu suffisamment d'informations pour faire une étude plus approfondie de ce dispositif...

10 Si les deux premières phrases sont impersonnelles, puisqu'il s'agit de rapporter un fait "extérieur», je apparait dès lors que l'expérience commence et qu'arrivent les complications ; je assume ainsi son histoire et ses aléas. À ce propos, on notera le recours à la désignation impersonnelle du ou des acteurs autres (« Il a été décidé, Il était prévu »), voire à leur effacement («j'ai au contraire été écartée »); il est évidemment difficile pour l'auteur de mentionner ces autres dans une histoire qui les voit surgir comme des opposants... surtout quand on les attendait comme adjuvants.

L'espace ${ }_{20}^{\circ}$ comme un autre double

11 Le MPU se situe dans un espace, comme dans un temps, nécessairement attesté (voir en [1] l'identification du lieu du stage). Ceci dit, cet espace, comme le sujet-actant et sans doute plus que le temps, fait l'objet d'une représentation puisqu'il est la scène des événements :

(5) Après l'état des lieux que nous avons dressé dans le chapitre précédent, il convient maintenant de s'interroger dans quelle mesure il est pertinent d'intégrer un centre de ressources dans cette école de langues privée.

Or si l'auteur peut faire allusion (comme au tout début de son mémoire, cf. (2)) à un contexte "large » et plutôt conceptuel, on voit par contre comment il vise ici, par cette référence démonstrative, une portion d'espace particulièrement définie (entre autres par rapport à soi) ; c'est une deuxième différence importante entre mémoire professionnel et mémoire scientifique. Et l'on peut penser que c'est dans cette double référence à un monde repéré par rapport à soi ("Mon stage, cette école ») et à un ensemble d'entités ou de notions certes spatialisées mais plus abstraites ("l'Europe, un monde qui se rapproche »), nécessaires l'un et l'autre au MPU, qu'opère la concurrence entre je, nous et on...

Nous allons maintenant consacrer notre deuxième partie à une étude pragmasémantique détaillée des emplois de ces marques personnelles, c'est-à-dire de leurs contextes et de leurs effets.

Pragmasémantique d'un MPUPrésentation des occurrences

Le MPU sur lequel nous travaillons comporte 32000 mots (environ 90 pages) dont :

15111 occurrences de la $1^{\text {re }}$ personne du singulier

$69 \mathrm{Je} / \mathrm{j}^{\prime}$ et $14 \mathrm{me} / \mathrm{m}^{\prime}$

28 mon, ma, mes

16120 occurrences de la $1^{\text {re }}$ personne du pluriel

nous : 83

notre, nos : 37 ,

17 soit une répartition à peu près égale. Les occurrences de on sont au nombre de 29 , donc bien moindres. 
Nous nous sommes fixé ici deux tâches :

- trier les deux types de nous, collectif $v s$ académique :

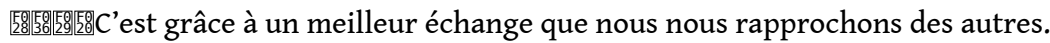

(7) Le fonctionnement du centre de ressources est soumis à certaines conditions [....]; nous les exposerons ici et reviendrons sur les différents points au cours de notre exposé.

- caractériser l'environnement actantiel de je et de nous : en tant que sujets ou objets de verbes d'action $v$ de perception $v s$ d'opinion $v s$ de parole.

Nous, l'académique et le collectif

En ce qui concerne la première tâche, il apparait clairement que les emplois ont des contextes, sémantiques ou référentiels, syntaxiques et pragmatiques, bien définis.

Le nous académique, que l'auteur (dans une note) appelle "rhétorique " ", apparait dans les emplois que l'on dira de gestion textuelle ou discursive. Dans ces cas, il est strictement équivalent (du point de vue référentiel) à je :

(8) C'est grâce à un meilleur échange que nous nous rapprochons des autres, mais cette nouvelle solidarité demande également plus de capacités de communication ;

Vs

(9) Dans un premier temps, c'est-à-dire dans le présent chapitre, nous allons poser la question...

On peut néanmoins en faire une autre interprétation: si l'auteur choisit cette forme, ce n'est pas seulement parce que, malgré ce qui est affirmé en note, nous est une forme conventionnelle de désignation de soi, mais bien parce que cette personne plurielle confère une "autoritét ». En somme, si, comme on le verra (section suivante), l'auteur entend bien assumer personnellement et individuellement ses responsabilités de stagiaire, et donc s'il va employer le je notamment pour le récit des moments les plus tendus de l'histoire vécue, il entend bien ici respecter le sérieux et la pompe de l'exercice académique. Mais il ne s'agit pas de pose, parce qu'il est demandé au candidat au diplôme de faire la preuve, par la gestion de son discours, de ses compétences générales en matière d'organisation, de planification. Le nous académique peut être interprété alors comme celui d'une communauté scientifique qui partage les mêmes conventions en matière d'exposition des savoirs.

Le nous collectif est employé lorsqu'il est fait référence (10) à un espace partagé (géographique ou social), ou bien (11) à la communauté professionnelle du stage, ou encore (12) à une communauté d'esprit qui peut être autre que (voire opposée à) la précédente :

(10) C'est grâce à un meilleur échange que nous nous rapprochons des autres, mais cette nouvelle solidarité demande également plus de capacités de communication.

(11) Investir dans ce nouvel outil [un centre de ressources], c'est faire le pari qu'il amène des avantages pour une formation dans un certain contexte donné. L'avantage espéré est dans notre cas de nature économique.

(12) [suite de 9] [Dans un premier temps, c'est-à-dire dans le présent chapitre, nous allons poser la question des apports que le multimédia peut avoir pour l'enseignement en langues] et l'utilité potentielle d'un centre de ressources multimédia pour NN Paris; les possibilités qu'offrent les NTF pour l'apprentissage sont néanmoins contraintes par le mode de formation qui les emploie 睗 notre souci primordial dans la définition de ce dernier sera de servir au mieux les besoins et souhaits individuels de l'apprenant.

Dans le dernier cas, il s'agit d'un emploi de connivence, qui vaut pour nous deux, c'est-àdire pour un je qui s'adresse à une deuxième personne (le lecteur-évaluateur), et qui instaure par là un rapport de complicité avec lui. On pourrait analyser alors, 
interactionnellement, la situation en termes de face et de hiérarchie (Maingueneau, 1998, notamment les ch. 2 et 3): l'auteur place le lecteur en position d'interlocuteur et de maitre ; il le replace dans son rôle de formateur, en (lui) rappelant (ou en faisant appel à) ses théories, ses enseignements (en l'occurrence sur les principes d'autoformation, l'individualisation des apprentissages...) :

(13) [extrait de 12] notre souci primordial sera de servir au mieux les besoins et souhaits individuels de l'apprenant.

Il s'agit donc ici d'un nous dialogique ou polyphonique, qui superpose les voix. D'une part, en ce qu'il introduit une forme de citation, en tout cas un emprunt de paroles et pensées :

(14) votre souci comme le mien (ou le mien à la suite du vôtre) est de servir...

D'autre part, en ce qu'il permet de fondre ces deux espaces discursifs et mentaux en un seul :

(15) nous pensons et proclamons en commun que servir...

Les exemples sont suffisamment nombreux dans ce MPU pour que nous y ayons prêté attention... Considérons les trois extraits suivants :

(16) Ainsi, nous poursuivons la finalité de créer le meilleur environnement possible à l'apprentissage des stagiaires à NN Paris. Nous recherchons ainsi à favoriser la meilleure adéquation entre leurs préférences et besoins d'un côté et leur formation d'un autre côté [...] Sera-t-il mieux/nécessaire que l'apprenant travaille de manière autonome ou plutôt guidée, avec plus ou moins d'initiatives ? Vaut-il mieux adopter le mode de l'hétéroformation ou de l'autoformation?

(17) Nous constatons en conclusion que l'individualisation est limitée dans un rapport d'hétéroformation. Et pourtant, la communication et la correction par un être humain sont indispensables à l'acquisition d'une langue étrangère...

(18) Comme la formation dispensée par un enseignant ne remplit pas pleinement nos exigences, c'est celle dirigée davantage par l'apprenant que nous revendiquerons en complément, c'est-à-dire l'autoformation.

Ces trois dernières occurrences sont très proches dans le texte et appartiennent toutes à une section intitulée Servir au mieux les besoins individuels des apprenants. Cette section est donc l'application du souci émis en (13), elle fait suite à l'état des lieux, elle lui sert de prolongement, elle consiste à envisager les préconisations. On voit donc tout l'intérêt qu'il y a pour le rédacteur de recourir à cette forme personnelle collective: non seulement le message de l'enseignant a été assimilé ou récupéré au premier contact avec le terrain (phase d'observation et de diagnostic), mais il est réinvesti dans la phase (projetée) de remédiation.

Je... dans le temps et l'actionSoi et les autres 嚂aux divers temps

27 Il est certain que pour caractériser le MPU comme genre d'écrit universitaire, le relevé des occurrences linguistiques de la première personne (singulière) se révèle un exercice fructueux; ne serait-ce que par leur nombre sans équivalent dans un mémoire de recherche scientifique. Analysons maintenant ses contextes et ses effets d'emploi.

À cet égard, le trait le plus marquant est que la première personne apparait quasi exclusivement dans les séquences narratives', en position sujet (ou objet) d'un verbe conjugué à un temps du passé, notamment au passé composé, dont on connait la valeur d'écho dans le présent du narrateur (voir l'enchaînement avec nous + présent en [21]) :

(19) Mon stage s'est déroulé de janvier à juin 1998.

(20) Par ailleurs, la formule avec les NTF [Nouvelles Technologies de Formation] ne rencontre pas un grand succès auprès des stagiaires jusqu'à présent, même si les 
formateurs m'ont fait part de la curiosité et de l'intérêt que les apprenants manifestent régulièrement en voyant le centre de ressources.

(21) La fréquentation trop faible du centre de ressources ne m'a pas permis d'établir un profil d'utilisateurs plus approfondi. Nous prenons donc ces indications comme données.

(22) Il était prévu que je sois intimement liée à ce projet, ce qui n'a en définitive pas été le cas ; j'ai au contraire été écartée de toutes les décisions qui ont été prises. Par ce fait, je n'ai malheureusement pas eu suffisamment d'informations pour faire une étude plus approfondie de ce dispositif et peux en juger seulement à travers les remarques de deux apprenants et du formateur qui a assuré la présence en centre de ressources à YY.

(23) Un apprenant au CNAM m'a fait part de son attirance vers le multimédia.

- lorsqu'il est académique, dans un énoncé au futur, mais assez souvent aussi à des temps proches du présent du moment de l'énonciation écrite (passé récent, futur proche), c'est-àdire à des temps qui permettent de coller au plus près au temps de cette énonciation, à son étalement, à sa durée, à son accomplissement : " nous allons, nous venons de ». Cette fréquence rejoint ce que nous avons pu dire du lien qui existe entre l'emploi de cette forme personnelle et sa fonction de gestion métadiscursive, c'est-à-dire de discours sur l'organisation de l'écrit en cours ;

- lorsqu'il est collectif, l'énoncé est au présent, un présent qu'on pourrait dire de longue durée, celui de la communauté partagée, qui elle-même s'inscrit dans des espaces-temps plus ou moins longs (la mondialisation, l'Europe). On se retrouvera aussi dans ce type d'environnement temporel, celui d'un présent étiré, même s'il n'est pas intemporel : (24) Suivant le niveau linguistique et les besoins de l'apprenant, les grandes lignes $\mathrm{du}$ contenu de la formation sont conçues. On détermine plus particulièrement quelles sont les choses que la personne doit voir et apprendre afin d'atteindre les finalités fixées.

(25) Avec ou sans NTF, les formations sont toutes conçues de la même manière. C'est-à-dire que l'on prévoit la formation " classique », et que l'utilisation du centre de ressources multimédia y est ajoutée si jamais le client l'a demandée.

(26) Le terme EAO (Enseignement Assisté par Ordinateur) parait aujourd'hui démodé, et on lui préfère généralement celui de NTF (Nouvelles Technologies pour la Formation), lorsque l'on parle des produits parmi les NTIC (Nouvelles Technologies de l'Information et de la Communication) qui sont destinés à l'apprentissage.

31 Mais on a une autre valeur sémantique et produit un autre effet : son emploi permet de donner à l'énoncé ou à l'assertion une valeur de vérité générale, ou plus générale, en tout cas peu susceptible de contestation (26) ; et de même que on dépersonnalise l'énonciation, le présent ainsi détemporalisé hausse la réflexion au niveau de l'abstraction scientifique :

(27) Si la relation entre stagiaire et formateur peut se transformer, c'est grâce à une nature différente de la formation avec ce support par rapport à la formation face à face.

De l'action au texte 
Pour continuer et finir, au travers de notre exemple, de caractériser le MPU en tant que genre, nous relèverons et commenterons maintenant les traits suivants :

- si je désigne, dans le récit notamment, un sujet acteur, agent ou actant, il est aussi la marque d'un sujet de parole et de conscience ;

- en ce qui concerne le sujet de parole ou parlant, nous ne reviendrons pas sur les emplois du nous métadiscursifs. Il nous semble plus intéressant de nous pencher ici sur des occurrences telles que : «je parle ici d'une autoformation, je le répète », c'est-à-dire sur la manière dont l'articulation entre l'action et la réflexion se fait dans le texte - ou plus exactement dont elle est faite par le texte : on trouve cette articulation, notamment, entre d'une part «j'ai conçu, j'ai réalisé », d'autre part « je ne le pense pas, je n'adhère pas à ce point de vue, j'entends, j'estime » (4 occurrences), « je constate ». Nous allons montrer le rôle médiateur que jouent la personne, le texte et singulièrement la narration dans ce dispositif?

Du côté de l'action, assumée et décrite à la première personne, on relève aussi bien des occurrences proprement agentives et narratives (telles que : «j'ai conçu, j’ai réalisé »), que des procès qui montrent le (réd)acteur dans des phases ou en position plus réflexives : «mes observations se limitent, je me suis concentrée». On peut supposer que cette position ou ces phases, notamment lorsqu'elles tendent à dépasser ou à gommer le cadre temporel du récit, précèdent, appellent même, des affirmations du type: "je pense, j'entends, j'estime, ou encore je rejoins en cela l'avis de ». Or ce qu'il est important de souligner, à ce moment-là, c'est que l'agent-personnage est aussi représenté narrativement dans des situations d'échange verbal : «j'ai proposé [à une personne] de choisir son logiciel, il m'importait d'avoir l'avis des utilisateurs sur ce point, j'ai posé les questions, j'ai voulu connaitre les impressions et les opinions ». Sans compter les cas où ce sont les paroles d'autrui qui sont rapportées, intégrées au récit au discours direct, où donc le narrateur est impliqué comme interlocuteur :

(28) La formatrice R. de NN Paris observe, en parlant d'un apprenant: «J'ai voulu lui montrer comment ça fonctionne, mais elle a l'habitude de l'ordinateur. Elle s'en sert beaucoup mieux que moi!»

(29) [...] la formatrice R. l'exprime ainsi : «Je pense que ça change par rapport au cours normal, avec le professeur, le tableau, le livre... C'est très intéressant [...] C'est une autre porte ".

La parole est ainsi mise en scène, sous diverses formes, mais notamment comme outil de connaissance, de conseil, d'orientation, de persuasion, donc d'exposition et d'argumentation. De la sorte, la représentation autobiographique du personnagenarrateur agissant par la parole participe à la mise en scène ou en intrigue de son histoire, dans des phases qui précèdent et appellent, on l'a dit, la réflexion. Dès lors et au-delà, ce dispositif permet, d'une part, de tisser la trame cohésive du récit dans sa dimension d'histoire de vie, de l'autre, d'assurer la pertinence du mémoire comme texte nécessairement ré-flexif. On comprend dans ces conditions que, lorsque le rédacteur du MPU que nous avons dépouillé est dans cette position ou à ce stade de la réflexion, il continue avec autant d'insistance et d'assurance de parler en son nom personnel : « je ne le pense pas, j'estime, je constate. » Bien sûr le nous serait alors toujours possible ; il n'en reste pas moins que dans ce texte (pour s'en tenir à une vérification rapide et facile à faire en traitement de texte), il y a trois occurrences de «à mon avis », une de "selon moi », et aucune avec notre ou nous. Je est donc bien le, sinon le seul, moyen de tisser efficacement et de manière cohérente le fil qui liera l'action au texte.

Prolongements 
Cette analyse linguistique énonciative pourrait être prolongée dans deux sens :

- en focalisant sur les séquences où alternent un je et un nous :

(30) Le public de NN Paris, et donc le public potentiel du centre de ressources, est notamment composé d'assistantes de direction, de cadres (dans les domaines juridique, informatique et financier) et de techniciens supérieurs (dans la télécommunication et l'électronique), d'après $\mathrm{M}$. $\mathrm{X}$. La fréquentation trop faible du centre de ressources ne m'a pas permis d'établir un profil d'utilisateurs plus approfondi. Nous prenons donc ces indications comme données.

- en étudiant les formes indéfinies, telles que on, mais aussi les disparitions du sujet ou de l'agent :

(31) L'objectif du stage a changé plusieurs fois, en raison d'un recadrement contextuel, et des besoins qui se sont présentés pendant son déroulement. Les activités effectives ont été les suivantes.

Conclusions et perspectives didactiques

À l'issue de cet essai de description du MPU, on peut retenir comme caractéristiques de ce genre les traits suivants :

- l'inscription dans l'histoire et le rôle joué par l'auteur : le MPU est aussi un récit, et un récit autobiographique. L'identité (donc la compétence) professionnelle à construire ou à justifier passe par un exercice de ré-flexion qui engage aussi l'auteur comme acteur (ce que nous tentons de signifier par réd-acteur).

- le MPU s'inscrit également dans un espace qui n'est pas seulement celui d'une discipline, d'une spécialité ; le MPU doit donc représenter des entités qui sont autres qu'abstraites. Il y a dans le MPU une relation au terrain et, en termes narratifs ou descriptifs, une relation du terrain qui le rapproche sans doute de la méthode ethnographique ou sociologique.

- plus près du texte et de l'énonciation, le rédacteur prend part comme acteur au récit, i. e. à l'exposition et l'ordonnancement des évènements ; $i$ (Page number) doit donc assumer en son nom propre un certain nombre de faits, et particulièrement porter sur son histoire et son environnement (réel et conceptuel) des jugements qui l'engagent directement.

Enfin, à un autre plan, didactique, il semble que la formation des étudiants à la rédaction d'un MPU passe par la prise de conscience et l'exercice d'un certain nombre de faits linguistiques ou textuels : la diversité des genres de textes et leur articulation; les plans d'organisation du texte (référentiel, énonciatif, thématique); la représentation des paroles et pensées, de soi et d'autrui, et les marques de la subjectivité dans le langage; l'organisation et les fonctions du récit, factuel et fictionnel; la place du personnage dans la représentation narrative ; la constitution de l'identité par la narration.

\section{BIBLIOGRAPHIE}

COHN, D. (2001) : Le propre de la fiction, Paris, Seuil (ch. 2 : Vies fictionnelles et vies historiques, 35-63).

DEMAIZIÈRE, F. et UEBERSFELD, J. (2002-2003) : Approche du mémoire professionnel universitaire, en ligne sur le site AEM : <http://didatic.net/article.php3?id_article=41>. 
DUCROT, O. (1989) : Logique, structure, énonciation, Paris, Éd. de Minuit.

MAINGUENEAU, D. (1998) : Analyser les textes de communication, Paris, Dunod.

\section{NOTES}

1. Suivant Benveniste (1966 et 1974), puis Kerbrat-Orecchioni (1980, rééd. 2002), Ducrot (1989) et Adam (1999).

2. Ce qui ne veut pas dire, automatiquement, une fiction (Cohn, 2001).

3. " L'emploi du je et du nous variera dans ce travail suivant la nature de l'énoncé. Le je remplacera le nous rhétorique lorsqu'il s'agira d'un point de vue personnel ou d'un fait concernant ma personne (comme le stage)».

4. « Avouerai-je enfin que je n'ai pas de règle fixe dans l'usage du « je » et du « nous », à l'exclusion du « nous » d'autorité et de majesté ? Je dis de préférence « je » quand j'assume un argument et « nous » quand j'espère entrainer à ma suite mon lecteur... » (Ricœur, 2000 : Avertissement, 3).

5. Des séquences parfois très réduites : voir (20).

6. Il s'agit là d'une référence à Ricœur (1986 ; voir aussi Demaizière et Uebersfeld, 2002).

7. En nous inspirant encore des ouvrages de Ricœur $(1986,1990)$.

\section{RÉSUMÉS}

Le mémoire professionnel universitaire (MPU) étant un outil de formation (et d'évaluation) à la fois théorique et pratique à BAC + 5 (ex-DESS, masters), son écriture est « hybride » du point de vue d'abord des marques d'énonciation. Ainsi l'alternance de je-nous singuliers et de nous pluriels est non seulement caractéristique en surface de ce (genre de) texte, mais elle éclaire en profondeur la figure de ce que nous nommerons un (réd)acteur, autrement dit d'un auteur qui est aussi le personnage-clé d'une expérience professionnelle, dont à la fois il fait le récit et tire les leçons. L'étude des marques d'ancrage spatio-temporel permet de confirmer et d'affiner cette analyse. 